\title{
Evaluation of Renal Disorders in Type 2 Diabetic Patients Using Ultrsonography
}

\author{
Saddig D. Jastaniah ${ }^{1}$, Naglaa M. Alsayed ${ }^{\mathbf{1}}$, Ibrahim A. Awad ${ }^{\mathbf{1}}$, \\ Hashim R. Fida ${ }^{2}$, Hammad H. Elniel ${ }^{1}$ \\ ${ }^{1}$ Diagnostic Radiology Department, King Abdulaziz University, Jeddah, Saudia Arabia \\ ${ }^{2}$ Family Medicine Department, King Abdulaziz University, Jeddah, Saudia Arabia \\ Email: sjastaniah@kau.edu.sa
}

Received November 23, 2013; revised December 10, 2013; accepted December 18, 2013

Copyright (c) 2013 Saddig D. Jastaniah et al. This is an open access article distributed under the Creative Commons Attribution License, which permits unrestricted use, distribution, and reproduction in any medium, provided the original work is properly cited.

\begin{abstract}
Type 2 diabetes mellitus (type $2 \mathrm{DM}$ ) is one of the common epidemics worldwide. The prevalence of type 2 DM is high among Saudi population. Renal affection by DM is in the form of nephropathy that may end in the end stage of renal disease (ESRD) and renal failure warrants chronic dialysis or renal transplantation. Renal Ultrasonography (US) is a simple, accurate, affordable and non-invasive test that can help in management of type 2 DM patients who have renal affection. Four hundred patients, aged from 13 - 93 years with type 2 DM were recruited from the outpatient internal and family medicine clinics at KAUH. Renal US was requested for 202 patients, and was done at the diagnostic radiology department using digital iU22 Philips machine with 3.5 - $5 \mathrm{MHz}$ convex array transducer. The renal size, parenchymal echogenicity and associated renal pathologies e.g. stones, cysts or masses were evaluated. Statistical analysis was performed using SPSS program version 16. Most cases showed normal renal size and echogenicity. Grade one nephropathy was more than grade two or three. Nephropathy was non-significantly correlated to the patients' gender, but to their age. Increased patients' age was associated with increased renal echogenicity. Non-diabetes-related renal abnormalities were detected in $39 \%$ of patients. The most common of non-diabetes-related renal abnormalities was simple renal cyst followed by renal stones (25\% and $23 \%$ ) respectively. Renal US for patients with type 2 DM has a great role in diagnosing and grading diabetic nephropathy, selecting cases with non-diabetic nephropathy for renal biopsy, and detecting associated renal abnormalities. Due to the high prevalence of DM in Saudi Arabia, we recommend future expanding study of the underlying possible genetic relation between DM and renal cysts and also the relation between renal stones and type 2 DM.
\end{abstract}

Keywords: Nephropathy; Ultrasound; Diabetic; Grade; Parenchyma

\section{Introduction}

Ultrasound has been routinely used for decades in the diagnosis of different renal diseases owing to its great advantages i.e. being non-invasive, reliable, widely available, and affordable. Although the kidney is always affected in the course of diabetes mellitus (DM), yet Type 2 diabetes has a more variable course. Patients often present at diagnosis with microalbuminuria due to delays in diagnosis and other factors affecting protein excretion. Some patients with microalbuminuria may suffer from renal complications. Reluctance in treatment, may lead overt nephropathy in about $30 \%$ of patients and, after 20 years of nephropathy, approximately $20 \%$ develop ESRD (end stage renal disease) [1]. The prevalence of DM in the Saudi population is high and $90 \%$ of diabetic have
Type patients II DM [2].

There are no data available on the incidence of diabetic renal disease in Saudi diabetics. However, the vast majority of Saudi diabetics entering dialysis (96\%) are of Type II [3].

A renal ultrasound is typically obtained to measure the renal size and echogenicity. Renal enlargement may be seen early in diabetes due to hyper filtration, while in late stages, the kidneys diminish in size from glomerulosclerosis. In addition, renal cortical hyperechogenicity is seen suggesting deteriorated renal function. Ultrasound is used also to exclude non diabetes-related renal disorders e.g. renal stones, masses or hydronephrosis [4].

Previous literature suggests that non-diabetic nephropathy complicating type 1 diabetes mellitus is rare, accounting for $2 \%-3 \%$ of diabetic patients with pro- 
teinuria. In contrast, non-diabetic kidney disease is a common finding in patients with type 2 diabetes mellitus. non-diabetic nephropathy was evident on kidney biopsy in about $22 \%$ of European and $26.7 \%$ of Asian patients with type 2 diabetes mellitus meaning that kidney biopsy may become a useful diagnostic option among those patients. However, renal biopsy cannot be used as a routine diagnostic test in all type 2 diabetic patients with proteinuria [5].

Renal Ultrasound can diagnose diabetes and non-diabetic-related nephropathy in type 2 diabetic patients. It can also detect non-diabetic-related structural abnormalities e.g. renal cyst, mass and stones. So, US can help early diagnosis and treatment of renal affection, reducing the progression of nephropathy and delaying or eliminating the need of dialysis or renal transplantation, and reducing the need of renal biopsy.

Aim of work:

The aim of this work was to study the role of US in the assessment and differentiation of kidney diseases in patients with type $2 \mathrm{DM}$.

\section{Patients and Methods}

\subsection{Patients}

This study included 400 type 2 diabetic patients ranging in age from 13 - 93 years. Patients were recruited from the outpatient internal and family medicine clinics at KAUH from 1/4/2012 to 1/4/2013 after obtaining an ethical approval from the ethical committee of medical researches. As renal Ultrasound was requested as a part of the routine management of type 2 diabetic patients, no written consent was required.

We classified the study population into four groups according to their age; patients less than 40 years (6 patients), from 40 - 50 years (29 patients), from 50 - 60 years (70 patients) and the largest group was found to be more than 60 years (97 patients). All patients were on regular follow up and had controlled diabetes.

\subsection{Methods}

Renal US was done for 202 patients (50.5\%) at the diagnostic radiology department, KAUH to measure the renal size, detect and grade renal parenchymal echogenicity, and to exclude the presence of associated renal disorders e.g. stones, masses or hydronephrosis. US was done using digital iU22 Philips real time machines with 5 - 1 $\mathrm{MHz}$ convex array transducers by expert sonographers and radiologists. US was done with the patient in the supine position. Axial and sagittal images were taken, the length of each kidney was measured in the sagittal plan. The renal parenchymal echogenicity was estimated compared to the hepatic and splenic echogenicities, and was classified into; grade 1 nephropathy, where the renal parenchymal echogenicity is equal to that of the liver or spleen, grade 2 nephropathy, where the renal parenchymal echogenicity is more than that of the liver or spleen, and grade 3 echogenicity where the renal parenchymal echogenicity is equal to the renal sinus fat. Associated renal diseases where fully evaluated. hydronephrosis was classified into mild, moderate or severe, and the level and cause of dilatation was studied. Renal cysts and masses were measured in 3 dimensions, and their echogenicity and vascularity were studied as well.

\subsection{Statistical Analysis}

Patients' demographic data, together with the US findings data were collected and statistically analyzed using using SPSS program (statistical package for social science) version 16. Qualitative data were presented in the form of number and percentage. Quantitative data were presented in the form of mean and standard deviation. Chi-square test was used for comparisons of qualitative data. Yates correction was made when it was indicated. Significance was considered when p value less than 0.05 .

\section{Results}

The population study included 400 patients with type 2 $\mathrm{DM}$, ranging in age from 13 - 93 years with the mean age is $58.86 \pm 12.98$. Females were more affected than males (59\%:41\%) respectively. renal Ultrasound was requested for $50.5 \%$ of the patients.

Regarding the renal parenchymal echogenicity, we found that most cases showed normal echogenicity (87.6\% on the right side and $88.1 \%$ on the left side), while grade 1 nephropathy was more common than grade or 3 "Figures 1(a) and (b)". Distribution of abnormal echogenicity is shown in "Table $\mathbf{1}$ ".

No statistically significant difference was found between different grades of echogenicity of the right kidney in both genders ( $p$ value $=0.21$ ), while on the left side, normal renal echogenicity was more in females and grades one and two nephropathy were more common in males $(p$ value $=0.048)$.

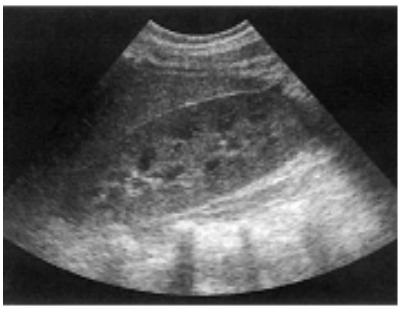

(a)

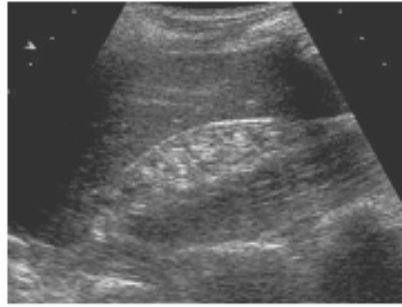

(b)
Figure 1. Abnormal renal parenchymal echogenicity. (a) Grade 1 nephropathy; (b) Grade 3 nephropathy (also notice the small renal size in (a) due to chronic nephropathy). 
Table 1. Renal parenchymal echgenicity for both sides $(n=$ 202 patients).

\begin{tabular}{lcc}
\hline Echogenicity (Rt. Kidney) & $\mathbf{N}$ & $\mathbf{( \% )}$ \\
\hline Normal & 177 & $(87.6)$ \\
Grade & 116 & $(7.9)$ \\
Grade & 26 & $(1.5)$ \\
Grade & 33 & $(0.7)$ \\
Echogenicity (Lt. kidney) & $\mathrm{N}$ & $(\%)$ \\
Normal & 178 & $(88.1)$ \\
Grade 1 & 16 & $(7.9)$ \\
Grade 2 & 6 & $(1.5)$ \\
Grade 3 & 2 & $(0.4)$ \\
\hline
\end{tabular}

We found that increased patients' age is associated with more affection with nephropathy (including the three grades) as seen in "Figures 2(a) and (b)".

We measured the renal size for all patients and we found it normal in most cases (98\% on the right side and $97 \%$ on the left side). Small sized kidney was detected in $2.5 \%$ on the right side and $1.5 \%$ on the left side. Only $0.5 \%$ of cases showed enlarged kidneys for each side. We found no statistically significant difference in the renal size of both kidneys in both genders ( $\mathrm{p}$ value $=0.25$ and 0.41 for the right and left sides respectively). Also, no statistically significance of the renal size abnormalities among different age groups was found ( $\mathrm{p}$ value $=$ 0.62 and 0.75 for the right and left sides respectively).

Associated renal abnormalities-not related to DMwere found in many cases. Some of them showed multiple abnormalities. "Table 2" showed the percentage of different non-diabetic related renal abnormalities among the study group.

Simple cortical renal cyst was found in 25 cases, right more than left (12 and 9 respectively), while 4 cases showed bilateral renal cysts "Figure 3".

Renal stones were found in 23 cases; left more than right (13 and 7 respectively). Three cases showed bilateral stones "Figure 4".

Hydronephrosis was found in 20 cases; 5 on the right, 10 on the left and 5 were bilateral "Figure 5".

Four cases showed solid renal mass; 2 on the right side, on the left side and one case had bilateral masses. We found no significant difference between male and female gender regarding the presence of associated renal pathology. Males were slightly more affected than females with renal cortical cysts, stones and hydronephrosis, while the females were more affected with renal masses and mixed findings with non-significant p value (0.166).

The distribution of different renal pathologies according to the patients' age is shown in "Table 3". No significant difference was detected between the studied age groups regarding presence of cyst, stone, hydronephrosis,

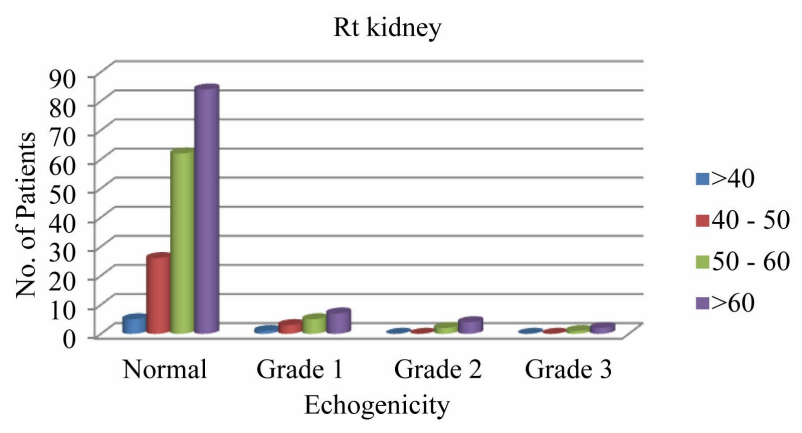

(a)

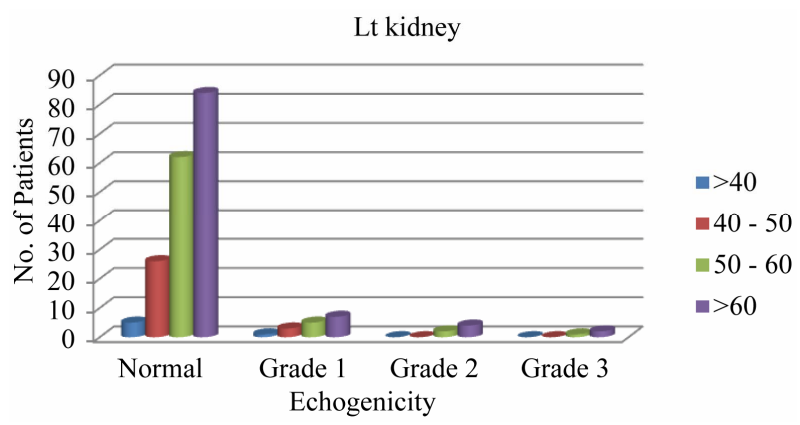

(b)

Figure 2. (a) The relation of different grades of increased renal echogenicity to different age groups on the right side; (b) The relation of different grades of increased renal echogenicity to different age groups on the left side.

Table 2. Distribution of non-diabetic related renal abnormalities among the study population.

\begin{tabular}{ccc}
\hline US finding & Number of patients & $(\%)$ \\
\hline Cyst & 25 & $(12.3)$ \\
Stone & 23 & $(11.3)$ \\
Hydronephrosis & 20 & $(9.9)$ \\
Mass & 4 & $(1.9)$ \\
Mixed findings & 7 & $(3.4)$ \\
\hline
\end{tabular}

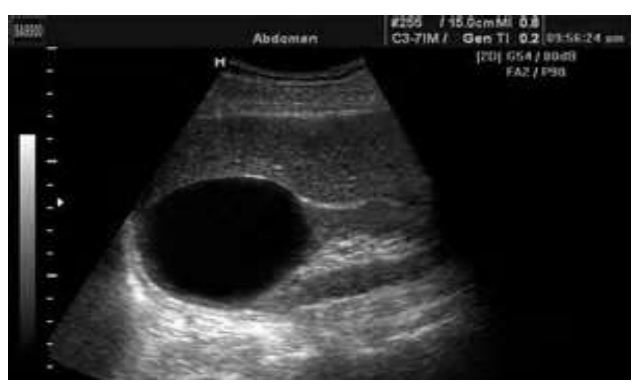

Figure 3. Shows the simple cortical renal cyst.

mass or mixed findings with the $\mathrm{p}$ value $=0.34$.

\section{Discussion}

Diabetes mellitus has become a worldwide epidemic, especially type 2 diabetes, which is expected to stand for 


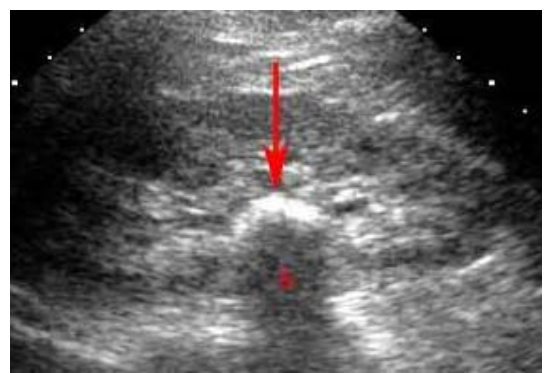

Figure 4. Sonographic image showing renal stone.

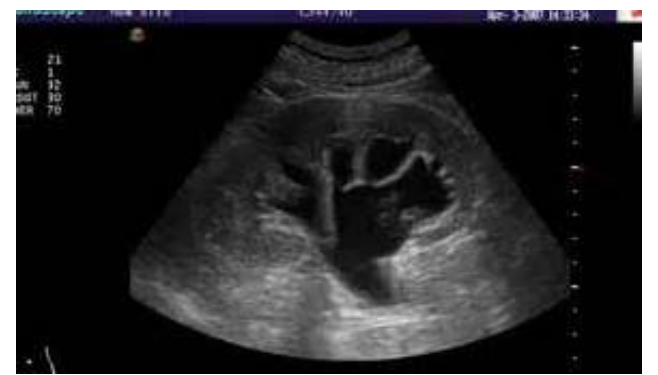

Figure 5. This shows hydronephrosis of the kidney.

Table 3. Deonstrates the ultrasound findings according to different age groups of the 202 patients.

\begin{tabular}{|c|c|c|c|c|c|c|c|c|}
\hline \multirow{4}{*}{ Ultrasound findings } & \multicolumn{8}{|c|}{ Age group } \\
\hline & \multicolumn{2}{|c|}{$<40 \mathrm{yrs}$} & \multicolumn{2}{|c|}{$40-50$ yrs } & \multicolumn{2}{|c|}{$50-60$ yrs } & \multicolumn{2}{|c|}{$>60 \mathrm{yrs}$} \\
\hline & No. of patients & 6 & No. of patients & 29 & No. of patients & 70 & No. of patients & 97 \\
\hline & Number & (\%) & Number & (\%) & Number & (\%) & Number & (\%) \\
\hline Cyst & 0 & 0 & 4 & 13.7 & 7 & 10 & 14 & 14.4 \\
\hline Stone & 0 & 0 & 1 & 3.4 & 7 & 10 & 15 & 15.4 \\
\hline Hydronephrosis & 1 & 1.6 & 3 & 10.3 & 3 & 4.2 & 12 & 12.3 \\
\hline Mass & 0 & 0 & 0 & 0 & 2 & 2.8 & 2 & 2 \\
\hline Mixed findings & 1 & 1.6 & 1 & 3.4 & 3 & 4.2 & 2 & 2 \\
\hline \multicolumn{9}{|c|}{$X^{2}=4.2, p=0.34$} \\
\hline
\end{tabular}

decades to come. In 2000, worldwide prevalence of diabetes was about 2.8\%. Between 2000 and 2030, the number of diabetic adults is expected to increase by $50 \%$ - $70 \%$ in developing countries and by $20 \%$ in developed countries [6]. In 2030, the prevalence of diabetes is expected to be $4.4 \%$ of the world population mostly related to the aging of people in the developed countries. The most important change in this prevalence appears to be an increase in the proportion of patients older than 65 years which was noticed in our study where $48 \%$ of patients were over 60 years [6].

Good primary and secondary prevention lead to decreased cardiovascular complications and increased life expectancy for patients with type 2 DMD. But this decrease in cardiovascular complications and death will lead to an increase in the incidence of ESRD among diabetic patients [6]. Diabetes mellitus has effects on most body organs and systems, one of which is the urinary system. Among the 202 patients in this study, 22\% showed different grades of nephropathy by US examination mostly grades one and two.

Diabetic nephropathy is the most frequent cause of terminal renal failure. So, once type $2 \mathrm{DM}$ is diagnosed, patients should be screened for diabetic kidney disease on regular basis. The screening includes urine and blood tests specific for renal functions [7]. In patients in whom microalbuminuria and/or proteinuria are found, measures should be taken to reduce cardiovascular risk and to slow down the progression of renal disease [7]. Ultrasound has a great role in diabetic patients with abnormal urine test results. It cannot only diagnose and grade nephropathy, but also exclude other renal diseases than diabetic nephropathy like chronic glomerulonephritis and ischaemic nephropathy [8]. Type $2 \mathrm{DM}$ is reportedly the leading cause of ESRD worldwide especially with longer duration of diabetes. However, non-diabetic renal diseases are not uncommon among T2DM patients with including acute interstitial nephritis, glomerulonephritides, hypertensive renal disease, and acute tubular necrosis which are potentially treatable $[5,9]$.

The prevalence of non-diabetic nephropathy among type 2 diabetic patients was evident on kidney biopsy. However, renal biopsy cannot be used as a routine in all patients with type 2 DM [5]. Renal ultrasound can be used as an adjunctive diagnostic method to select cases for biopsy. It is an easy, accurate, non-expensive and non-invasive test that can be used in diabetic patients to prove or exclude diabetic or non-diabetic nephropathy and also to diagnose associated renal diseases not related to DM e.g. renal cysts, stones or masses. In addition, Ultrasonographic evaluation of the kidney size is used to assess the progression of nephropathy [9]. Renal size measurement is an indicator to chronic renal failure and can be easily assessed using US in the sagittal plan. In our study, most patients had normal sized kidneys, while only $2.5 \%$ and $1.5 \%$ showed small kidneys $-<9 \mathrm{~cm}$ in length — on the right and left sides respectively. Small kidneys were associated with grade 2 or 3 nephropathy 
denoting underlying chronic renal failure.

Chong YB ET AL, 2012 studied the difference in the renal findings detected by US in type 2 diabetic patients with or without chronic renal failure nephropathy. They found that most of type 2 diabetic patients with chronic renal failure had small kidneys ( $<9$ cm length) [9].

Renal US examination is also helpful if the renal size is normal or increased and kidneys are symmetrical, as diabetic glomerulosclerosis is likely [10,11]. In our study, only $1 \%$ of patients showed enlarged kidneys $(>12 \mathrm{~cm}$ length) with normal echogenicity. Associated renal diseases not related to DM could also be assessed using US.

In the study of Chong YB ET AL, 2012 on type 2 DM patients, $15 \%$ of patients with chronic renal failure had associated simple cortical renal cyst, and $8 \%$ of patients with no renal failure had renal cysts [9]. In our study, $12.3 \%$ of all diabetic patients had simple cortical renal cysts. The relation between renal cysts and DM has been studied. Some literature proved it to be a coincidence, while others referred this to possible underlying genetic factors. Mutations in the gene encoding the transcription factor hepatocyte nuclear factor (HNF)- $1 \beta$ have recently been described in association with a variety of renal development abnormalities. The most consistent clinical feature is the presence of renal cysts and most affected subjects also have early-onset diabetes. The association of renal cysts and diabetes with an HNF- $\beta$ mutation is termed the renal cysts and diabetes (RCAD) syndrome Mutations in the HNF- $1 \beta$ gene may, therefore, be considered to cause a multisystem disorder. It is associated with pancreatic atrophy which explains the affection of those patients with DM between 10 - 61 (mean 26 years) [12]. Ultrasound can also accurately diagnose the presence of renal stones and hydronephrosis. In the current study 23 (11.3\%) patients had renal stones; either bilateral or unilateral, and 20 (9.9\%) had hydronephrosis which means that patients with type 2DM are subjected to renal stones more than normal population. it was proved that Kidney stones affect up to $5 \%$ of the population, with a lifetime risk of passing a kidney stone of about $8 \%-10 \%$ [13]. Our findings match with other literatures who found that type 2 diabetes is significantly associated with an increased risk for urinary stone formation. Type $2 \mathrm{DM}$ leads to lowering urine $\mathrm{pH}$ which is the main factor of uric acid (UA) stone formation. So, it was hypothesized that type 2 diabetes should favor the formation of UA stones. These findings suggest that UA nephrolithiasis should be considered as possibly reflecting a state of insulin resistance rather than simply UA stone formation. Accordingly, onset of UA nephrolithiasis in a patient should prompt a check for type 2 diabetes, especially in overweight patients [14]. Incidence of urinary stone disease rose considerably in recent decades in all industrialized countries $[15,16]$. As did the incidence of obesity, the metabolic syndrome, and type 2 diabetes $[17,18]$. These epidemiologic changes took place in parallel with marked changes in dietary habits and lifestyle that occurred in all Western and westernized populations, characterized by a high calorie intake together with reduced physical activity $[19,20]$. This temporal parallelism suggested that an association might exist among diabetes, obesity, and urinary stone disease. Conclusion and recommendation: renal Ultrasound for patients with type $2 \mathrm{DM}$ has a great role in diagnosing and grading diabetic nephropathy, selecting cases with non-diabetic nephropathy for renal biopsy, and detecting associated renal abnormalities not related to DM e.g. renal cysts and stones which allow to study the relation between them. Due to the high prevalence of DM in Saudi Arabia and the national efforts to reduce the incidence and complications of this epidemic, we recommend future expanding study of the underlying possible genetic relation between DM and renal cysts and also the relation between renal stones and type $2 \mathrm{DM}$.

\section{Acknowledgements}

This project was funded by the Deanship of Scientific Research (D S R), King Abdulaziz University, Jeddah, under grant no. (456/142/1433). The authors, therefore, acknowledge with thanks DSR technical and financial support.

\section{REFERENCES}

[1] P. M. Hall, "Prevention of Progression in Diabetic Nephropathy,” Diabetes Spectrum, Vol. 19, No. 1, 2006, pp. 18-24. http://dx.doi.org/10.2337/diaspect.19.1.18

[2] A. A. Al-Khader, "Impact of Diabetes in Renal Diseases in Saudi Arabia," Nephrology Dialysis Transplantation, Vol. 16, No. 11, 2001, pp. 2132-2135.

http://dx.doi.org/10.1093/ndt/16.11.2132

[3] A. Al-Zaid, S. Sobki and V. De Silva, "Prevalence of Microalbuminuria in Saudi Arabians with Non-Insulin-Dependent Diabetes Mellitus-A Clinic Based Study,” Diabetes Research and Clinical Practice, Vol. 26, No. 2, 1994, pp. 115-120.

http://dx.doi.org/10.1016/0168-8227(94)90148-1

[4] "National Kidney Foundation: Diabetes and Kidney Disease,” 2008.

http://www.kidney.org/atoz/atozItem.cfm?id=37

[5] E. Zukowska-Szczechowska and M. Tomaszewski, "Renal Affection in Patients with Diabetes Mellitus Is Not Always Caused by Diabetic Nephropathy," Roczniki Akademii Medycznej w Bialymstoku, Vol. 49, 2004, pp. 185189.

[6] C. Couchoud and E. Villar, "End-Stage Renal Disease Epidemic in Diabetics: Is There Light at the End of the Tunnel?” Nephrology Dialysis Transplantation, Vol. 28, No. 5, 2013, pp. 1073-1076.

http://dx.doi.org/10.1093/ndt/gfs559 
[7] V. Tesar, "Examination of the Kidneys in a Diabetic Patient," Vnitrni Lékarství, Vol. 54, No. 5, 2008, pp. 494498.

[8] E. Esmatjes, C. Castell, T. Gonzalez, R. Tresserras and G. Lloveras, "Epidemiology of Renal Involvement in Type II Diabetics (NIDDM) in Catalonia, the Catalan Diabetic Nephropathy Study Group,” Diabetes Research and Clinical Practice, Vol. 32, No. 3, 1996, pp. 157-163. http://dx.doi.org/10.1016/0168-8227(96)01256-9

[9] Y. B. Chong, T. C. Keng, L. P. Tan, K. P. Ng, W. Y. Kong, C. M. Wong, P. L. Cheah, L. M. Looi and S. Y. Tan, "Clinical Predictors of Non-Diabetic Renal Disease and Role of Renal Biopsy in Diabetic Patients with Renal Involvement: A Single Centre Review,” Renal Fail, Vol. 34, No. 3, 2012, pp. 323-328. http://dx.doi.org/10.3109/0886022X.2011.647302

[10] K. R. Tuttle, J. H. Stein and R. A. deFronzo, "The Natural History of Diabetic Nephropathy,” Seminars in Nephrology, Vol. 10, 1990, pp. 184-193.

[11] B. Brooks, R. Chong, I. Ho, F. Capstick, L. Molyneaux, T. T. Oo, et al., "Diabetic Retinopathy and Nephropathy in Fiji: Comparison with Data from an Australian Diabetes Center," Australian and New Zealand Journal of Ophthalmology, Vol. 27, No. 1, 1999, pp. 9-13. http://dx.doi.org/10.1046/j.1440-1606.1999.00146.x

[12] C. Bingham and A. T. Hattersley, "Renal Cysts and Diabetes 3.5-Syndrome Resulting from Mutations in Hepatocyte Nuclear Factor-1beta," Nephrology Dialysis Transplantation, Vol. 19, No. 11, 2004, pp. 2703-2708. http://dx.doi.org/10.1093/ndt/gfh348

[13] M. S. Parmar, “Kidney Stones,” BMJ, Vol. 328, No. 7453, 2004, pp. 1420-1424. http://dx.doi.org/10.1136/bmj.328.7453.1420
[14] M. Daudon, O. Traxer, P. Conort, B. Lacour and P. Jungers, "Type 2 Diabetes Increases the Risk for Uric Acid Stones," Journal of the American Society of Nephrology, Vol. 17, No. 7, 2006, pp. 2026-2033. http://dx.doi.org/10.1681/ASN.2006030262

[15] A. Trinchieri, F. Coppi, E. Montanari, A. Del Nero, G. Zanetti and E. Pisani, "Increase in the Prevalence of Symptomatic Upper Urinary Tract Stones during the Last Ten Years,” European Urology, Vol. 37, 2000, pp. 23-25. http://dx.doi.org/10.1159/000020094

[16] K. K. Stamatelou, M. E. Francis, C. A. Jones, L. M. Nyberg and G. C. Curhan, "Time Trends in Reported Prevalence of Kidney Stones in the United States: 1976-1994," Kidney International, Vol. 63, 2003, pp. 1817-1823. http://dx.doi.org/10.1046/j.1523-1755.2003.00917.x

[17] K. M. Flegal, M. D. Carroll, C. L. Ogden and C. L. Johnson, "Prevalence and Trends in Obesity among US Adults, 1999-2000,” JAMA, Vol. 288, No. 14, 2002, pp. 17231727. http://dx.doi.org/10.1001/jama.288.14.1723

[18] S. Wild, G. Roglic, A. Green, R. Sicree and H. King, "Global Prevalence of Diabetes: Estimates for the Year 2000 and Projections for 2030,” Diabetes Care, Vol. 27, No. 5, 2004, pp. 1047-1053. http://dx.doi.org/10.2337/diacare.27.5.1047

[19] S. J. Nielsen and B. M. Popkin, "Patterns and Trends in Food Portion Sizes, 1977-1998,” JAMA, Vol. 289, No. 4, 2003, pp. 450-453. http://dx.doi.org/10.1001/jama.289.4.450

[20] L. Cordain, S. B. Eaton, A. Sebastian, N. Mann, S. Lindeberg, B. A. Watkins, J. H. O’Keefe and J. Brand-Miller, "Origins and Evolution of the Western Diet: Health Implications for the 21st Century,” American Journal of Clinical Nutrition, Vol. 81, No. 2, 2005, pp. 341-354. 